\title{
II. EUROPA IN WEIMAR: \\ SENSIBILISIERUNG ZWISCHEN VERSAILLES UND GENF
}

Der Europadiskurs des Exils skizzierte Modellentwürfe politisch motivierter Gruppen und Individuen für eine spätere Epoche, ein Europe pensée für eine ferne Zukunft. Dabei griffen sie auf die Erinnerung an die häufig persönlich miterlebten Europabestrebungen in der Zwischenkriegszeit zurück - die optimistisch stimmenden Ansätze eines Europe vécue ebenso wie deren deprimierende Zerstörung zwischen den Nationalismen und Uneinsichtigkeiten der Jahre 1918/19 bis 1933. Diese Sensibilisierung für ein zumeist nur unscharf skizziertes Europa der Zukunft befand sich nach 1933 in Form persönlicher Wahrnehmungen, Reflexionen, Enttäuschungen und Hoffnungen im dürftigen Gepäck der Emigranten. Die in der Emigration häufig verbalisierte Enttäuschung über das Versagen des Völkerbundes als Friedensgarant und überstaatlicher Schlichter und die Philippiken gegen das Vertragswerk von Versailles zeigen, daß „Europabewußtsein häufig auch erst als Folge konkreter Einigungsresultate"1 - oder eben auch gescheiterter Einigungsbemühungen entsteht. Nahezu alle im Exil ausgearbeiteten Europakonzepte enthalten Details, die unmittelbar aus der Kenntnis tatsächlich vorhandener Institutionen und den realen Erfahrungen menschlicher und politischer Unzulänglichkeiten erwachsen sind 2 .

Die Sensibilisierung für den Europagedanken wurde in der Zwischenkriegszeit entwickelt, deren realpolitische Ansätze die späteren Emigranten weitaus nachhaltiger geprägt haben als der zunehmend isolierte Diskurs über das Ideal eines gemeinsamen Europas ${ }^{3}$. Im Exil erwiesen sich primär der Versailler Vertrag und der Völkerbund nicht nur als ständige Diskursbegleiter, sondern auch als häufig genutzte Negativfolien für modifizierte oder alternative Europakonzepte.

Im folgenden soll der Bewußtseins- und Erfahrungshorizont des Europadiskurses im Exil skizziert werden, soweit dies für das Verständnis der Quellenanalyse notwendig ist. Dabei wird das Kriegsende 1918 als geistes- und

\footnotetext{
1 Bosmans, Einleitung, in: Ders. (Hg.), Europagedanke 1996, S. 7.

2 Die parteipolitischen und programmatischen Stellungnahmen aus der Weimarer Zeit werden eingehend bei der Erörterung der Europabilder im Exil 1933-1937 berücksichtigt (Kapitel II. 2.). Zu den Europavorstellungen der Schriftsteller siehe die umfassende Studie von LÜTZELER, Schriftsteller und Europa 1992, insb. S. $312 \mathrm{ff}$. Die publizistische Debatte in den zeitgenössischen Zeitschriften wird eingehend behandelt in GRUNEWALD, BOCK (Hg.), Le discours européen (1918-1933) 1997.

${ }^{3}$ KRÜGER, Ansätze zu einer europäischen Wirtschaftsgemeinschaft 1984, S. 149 betont zurecht: „Indem man aber das übernationale Ideal und die politische Zielvorstellung zur Basis nahm, berührte man den eigentlichen Beitrag der Weimarer Republik zur Grundlegung einer europäischen Staatengemeinschaft kaum.“
} 
völkerrechtshistorische Zäsur erläutert und die daran anknüpfenden realpolitischen Ansätze in den Wahrnehmungsfeldern (Regierungs)Politik, Wirtschaft und Gesellschaft - gleichsam als Europe vécue der Zwischenkriegszeit - nachgezeichnet. Selbstverständlich sind die drei Wahrnehmungsfelder nicht voneinander zu trennen, sondern in ihrer historischen Interdependenz zu betrachten: "Il ne fait aucun doute que le mouvement vers l'intégration économique de l'Europe au $\mathrm{XX}^{\mathrm{e}}$ siècle ne peut être séparé de son contexte politique général et que les interactions sont nombreuses dans cette marche en avant entre les champs politique, culturel et économique. ${ }^{* 4}$

\section{Die historische Zäsur - der Erste Weltkrieg}

Der prominente Emigrant Heinrich Mann, Identifikationsfigur des literarischen Exils, legte in seinem einzigen im Exil beendeten Werk „Henri Quatre“ dem Titelhelden die selbstlos und weitblickend klingenden Worte in den Mund:

„Der Friede ist seinen Preis wert, wenn nicht wir allein ihn tragen, sondern alle christlichen Staaten überein. Ich und meine Verbündeten werden alle überzeugen, wo ihr Vorteil und ihre Sicherheit sind. Nirgends sonst, als in einem Völkerbund. “5

Mann, der sich mehrfach auch über die künftige Ordnung des europäischen Kontinents äußerte ${ }^{6}$, suggeriert hier eine geistesgeschichtliche Kontinuität europäischer Zusammengehörigkeit und Völkerverständigung, die bis heute in keiner Veröffentlichung zum Europagedanken unerwähnt bleibt. Doch handelt es sich dabei um ein letztlich geistiges Konstrukt, denn die Motivationen, die 1610 den "Grand Dessein“ des Herzogs von Sully trugen, erfuhren mit dem Ende des Ersten Weltkriegs eine solch tiefgreifende, revolutionäre Änderung, daß von einer ideengeschichtlichen Kontinuität kaum mehr gesprochen werden kann.

Die von Heinrich Mann intendierte Idealisierung sowohl der Herrscherpersönlichkeit Heinrichs IV. als auch des "Grand Dessein" war gemeint als politisch-literarisches Manifest für ein anderes als das von Mann erlebte Europa, ein Gegenentwurf zur realen Welt ${ }^{7}$. In einem Brief an Carl Stern-

4 BUSSIÈrE, Les milieux économiques 1997, S. 5.

5 MANN, Henri Quatre o. J., S. 850.

6 Vgl. dazu die Dissertation von KEIM, Europagedanke Heinrich Manns 1989, die stellenweise mit einer überschießenden Innentendenz behaftet ist und den eher vagen Europavorstellungen Manns die höheren Weihen einer durchdachten Gesamtkonzeption zu verleihen versucht.

7 Vgl. die literaturwissenschaftliche Exegese von HAHN, Heinrich Manns „Henri Quatre" 1981. Für den heutigen Betrachter gewinnt Hahns Darstellung vor allem durch die Kenntnis der auch politisch intendierten Diskrepanz zwischen der in der DDR praktizierten Staatsverehrung Heinrich Manns einerseits und der auch politisch motivierten westdeutschen Ignoranz gegenüber dem Werk des älteren Mann-Bruders andererseits. 
heim vom 29. Mai 1937 stimmte Heinrich Mann dem Urteil seines Bruders Thomas zu, „Henri Quatre“ sei „Synthese und Résuméc seiner ganzen Lebenserfahrung: „In diesem König hatte ich, gleich bei der ersten Bekanntschaft, die glänzendsten Anlagen gefühlt, wußte aber noch nicht, daß er mitsamt seiner Welt sich so herauswachsen würde. Jetzt paßt ihm jeder Gedanke, der mich bewegt, und mit dem Alter, seinem und meinem, stimmt es immer besser." ${ }^{8}$ Dem eigentlichen Verfasser des "Grand Dessein“, dem Herzog von Sully, ging es tatsächlich um die Lösung der beiden damals zentralen machtpolitischen Probleme9: die Beseitigung der Übermacht Habsburgs in Europa durch deren Verdrängung nach Lateinamerika und Asien sowie die Handhabung der Kirchenspaltung durch die Anerkennung der drei Konfessionen und eine strenge Umsetzung des Grundsatzes cuius regio eius religio. Wenngleich der Sully-Plan jeden Europa-Enthusiasmus als völlig zeituntypisch vermissen läßt, liegt seine übergeordnete Bedeutung dennoch in der Tatsache, daß der "Grand Dessein“ neben dem fast 140 Jahre zuvor erschienenen Plan Dubois' zu den ersten europäischen Entwürfen zählt, die im Zeichen des entstehenden Souveränitätsgedankens geschrieben wurden.

Deutlich visionärer als Sullys pragmatische Überlegungen nimmt sich das umfassende Reformwerk Pierre Dubois' aus dem Jahre 1306 aus $^{10}$. Unter dem Eindruck der Auseinandersetzungen französischer Kronjuristen mit Papst Bonifaz VIII. um den in der Bulle Unam Sanctam dogmatisierten päpstlichen Weltherrschaftsanspruch verlangte Dubois die politische Einheit der Katholiken unter Einschluß des byzantinischen Kaisers zur Vorbereitung eines Kreuzzuges in das seit 1291 verlorene Heilige Land. Das zu diesem Zweck einzuberufende Fürstenkonzil sollte nach Dubois als Kern eines christlichen Bundes gleichberechtigter Fürsten dienen, der die Einheit der Christenheit sowohl nach außen als auch nach innen gegen bellizistische Mitglieder zu schützen habe. Wesentlich war für Dubois der Konzilsgedanke, den er mit dem Vorschlag verband, den Papst gegen Entgelt unter die Oberherrschaft des französischen Königs zu stellen, um ihm die Konzentration auf die wahrhaft geistigen Aufgaben zu ermöglichen. Doch zielte die von Dubois konzipierte Recuperatione Terre Sancte eben nicht auf die Veränderung der bestehenden Welt, sondern die Errichtung einer neuen Welt, wodurch die Utopie seiner übergeordneten Zielsetzung unterstrichen wird ${ }^{11}$.

8 Zit. n. ibid. S. 463.

9 Zu Sullys Ansatz vgl. insbesondere MaLETTKe, Konzeptionen kollektiver Sicherheit 1992 sowie FOERSTER, Europa 1967, S. 131-143.

$10 \mathrm{Vgl}$. dazu den unverändert aufschlußreichen Aufsatz von OEXLE, Utopisches Denken 1977 sowie die Überblicksdarstellung bei FOERSTER, Europa 1967, S. 60-75.

11 OeXle, Utopisches Denken 1977, S. 339 bemerkt hierzu: „Aber bei allem Staats-Optimismus, der Dubois im französischen Staat seiner Zeit ein Instrument zur Verwirklichung seiner Vorschläge sehen ließ, sollte doch dieser Staat keineswegs auch der Ort sein, an dem er seine Vorschläge verwirklicht sehen wollte. Dafür war vielmehr ein neuer Ort, die Terra Sancta bestimmt. [...] Gleichwohl darf nicht übersehen werden, daß die Terra Sancta des Dubois außerhalb der res publica christiana, außerhalb der Ge- 
In den folgenden Jahrhunderten wurden der Öffentlichkeit immer wieder Pläne für eine Einigung Europas vorgestellt ${ }^{12}$. Besondere Beachtung verdienen die Überlegungen von Comenius (1645) ${ }^{13}$, William Penn (1693) ${ }^{14}$, John Bellers (1710) ${ }^{15}$, Saint-Pierre (1712) ${ }^{16}$, dessen „Mémoires“ Jean Jacques Rousseau 1761 wieder aufgriff, Immanuel Kant (1795) $)^{17}$, François de Paoli Chagny ${ }^{18}(1813)$ und Pierre Joseph Proudhon (1863) ${ }^{19}$. Gemeinsam war all

meinschaft christlicher Staaten und Völker lag. Vielleicht war es dies, was die Terra Sancta im Denken des Pierre Dubois zu einem Wunschtraum hat werden lassen. “

12 Einen unverändert lehrreichen und lesenswerten Überblick aus der Emigrationszeit bietet WeHBERG, Ideen und Projekte betreffend die Vereinigten Staaten von Europa in den letzten 100 Jahren, in: Friedenswarte, 41. Jg. (1941), S. $49 \mathrm{ff}$.

${ }_{13}$ Der tschechische Pädagoge Amos Comenius (Johan Amos Komensky) schlug 1645 auf der Grundlage seines pansophischen Ansatzes einer Allweisheitslehre einen Weltbund der Christenheit vor, bestehend aus drei Teilbereichen: Kultur, Politik, Religion, „eine woblgeordnete, durch gleiche Bande gleichen Wissens, gleicher Gesetze und wabrer Religion verbundene Gemeinschaft", zit. n. FOERSTER, Europa 1967, S. 162.

14 PENN, Essay toward the Present and Future Peace of Europe, London 1693. Der Quäker kam nach seinen Erfahrungen als Gründer und erster Gouverneur von Pennsylvania zu dem Schluss, nur ein auf gemeinsamem Recht basierender Bund der Völker, die in einem Bundesparlament mit staatseinkommensabhängiger Stimmgewichtung vertreten sein sollten, könne den Frieden dauerhaft gewähren. Auch Penns Entwurf beruhte - von einem Quäker nicht anders zu erwarten - auf dem Wunsch, das Christentum zu stärken. Doch übertrug er - unter dem Einfluss seines Freundes John Locke erstmals die Idee eines einheitlichen Rechts auf die Ebene der internationalen Beziehungen; vgl. FOERSTER, Europa 1967, S. 163-170.

15 BELLERS, Some Reasons for a European State, proposed to the Powers of Europe, by an Universal Guarantee, and an Annual Congress, Senate, Dyet, or Parliament, to settle any disputes about the bounds and rights of Princes and States hereafter, London 1710. Bellers bezog sich sowohl auf Sullys "Grand Dessein" als auch auf Penns Ansatz, wobei er unter Beibehaltung der bisherigen Staatengrenzen die Aufteilung Europas in 100 Kantone vorschlug, die je einen Delegierten in ein europäisches Parlament senden; vgl. FOERSTER, Europa 1967, S. 330 f. Bemerkenswert an Bellers Entwurf ist vor allem seine Rezeption durch den Sozialdemokraten Eduard BERNSTEIN, der in seiner noch während des Ersten Weltkrieges erschienenen Schrift "Völkerbund oder Staatenbund" 21919 für sich beansprucht, John Bellers als erster wiederentdeckt zu haben (S. 5 f.).

16 Mémoires pour rendre la Paix perpétuelle en Europe (anonym). Saint-Pierre führte in seinem berühmten Vorschlag eines europäischen Fürstenbundes erstmals das Prinzip der Mehrheitsentscheidung ein; vgl. FOERSTER, Europa 1967, S. 173-186. 1761 widmete sich Jean Jacques Rousseau dem Werk Saint-Pierres und überarbeitete dessen Europaplan unter Einfügung zahlreicher eigener Gedanken.

17 KANT, Zum Ewigen Frieden. Mit dieser noch vom deutschen Exil 1933-1945 vielfach zitierten Schrift leitete Kant die Wende von zumeist akademischen Utopien eines europäischen Staatenbundes zu einem an den konkreten Realisierungsmöglichkeiten orientierten Ansatz ein. Wesentliche Voraussetzung jeder europäischen Föderation war für Kant das republikanische Ordnungsprinzip; vgl. FOERSTER, Europa 1967, S. 209-219. 18 Paoli-Chagnys Entwurf für drei Staatenbünde - eine internationale „Föderation der Monarchen" unter dem Ehrenvorsitz des preußischen Königs sowie zwei nachgeordnete Bünde deutscher Staaten unter dem Protektorat des Zaren bzw. italienischer Staaten unter dem Protektorat des österreichischen Kaisers - wurde dem Wiener Kongreß vorgelegt; vgl. FOERSTER, Europa 1967, S. 343 f.

19 Proudhon, Du principe fédératif et de la nécéssité de reconstituer le parti de la ré- 
diesen Entwürfen, daß sie entweder auf die Stärkung der Christenheit und die Überwindung der dogmatischen Kirchenspaltung abzielten oder die Errichtung bzw. Manifestierung einer europäischen Hegemoniemacht im Blick hatten. Des öfteren diente die Idee des Heiligen Römischen Reiches Deutscher Nation als Folie20. Noch während des Ersten Weltkrieges als dem Höhe- und Wendepunkt des europäischen Imperialismus stand der Europagedanke im Zentrum diverser nationaler Hegemonialpläne, die im Deutschen Reich durch zahlreiche Mitteleuropa-Konzeptionen repräsentiert wurden ${ }^{21}$ und in dem 1915 erschienenen Werk von Friedrich Naumann ${ }^{22}$ eine primär ökonomisch motivierte Deutung erfuhren ${ }^{23}$.

Mit dem Ende des Ersten Weltkrieges trat in der ideen- und völkerrechtshistorischen Entwicklung des Europagedankens ein fundamentaler Wandel ein, der die europäische Motivationslage grundlegend ändern und in ein völlig neues Bedingungsfeld stellen sollte. Dieser Umbruch sei mit der Benennung der fünf ausschlaggebenden Faktoren kurz zusammengefaßt: das Ausmaß von Gewalt und Leiden, bedingt durch den technisch-militärischen Fortschritt; die weltwirtschaftliche Abhängigkeit Europas von den USA und das allgemeine Phänomen zunehmender weltwirtschaftlicher Interdependenz; Europas beginnender Niedergang als Machtzentrum der Weltpolitik; die faktische Überwindung der nationalstaatlichen Souveränität durch die weltpolitische Dichotomie und die im Gegenzug einsetzende emotionale Überhöhung der Nation.

War der Verlust der wirtschaftlichen Vorreiterrolle Europas schon seit längerem prognostiziert worden, so überraschte der Machtverlust in der Weltpolitik die Zeitgenossen in einem solchen Ausmaß, daß kaum jemand diese Konsequenz des Weltkrieges als grundlegenden Einschnitt hinzunehmen bereit war. Europa konnte nicht einmal seine eigenen Angelegenheiten mehr ohne außereuropäische Hilfe lösen und hatte erst recht die Kompetenz zur Lösung weltpolitischer Konflikte eingebüßt. Als das alte Europäische Konzert zerbrach, veränderten sich grundlegend auch die Positionen Großbritanniens und Frankreichs und schwächten ihre Bedeutung im globalen

volution, Paris 1863. Proudhons Anliegen bestand vor allem in der Verhinderung einer monarchistischen Restauration, die er durch die Aufteilung aller europäischen Staaten in Klein- und Kleinstföderationen unter dem gemeinsamen Dach einer „confédération des fédérations “ zu verwirklichen glaubte; vgl. BülCK, Föderalismus 1964, S. 13 f., insb. Anm. 34. In der Nachfolge Proudhons entwickelte der französische Jurist George Scelle im 20. Jahrhundert seine Theorie der Internationalen Organisationen.

20 Zum Heiligen Römischen Reich als europäischem Ordnungsprinzip: HammERSTEIN, Heiliges Römisches Reich deutscher Nation und Europa 1992.

21 Zur „Weltreichtheorie“ eines vereinten Mitteleuropas unter deutscher Führung vgl. UllRICH, Polnische Frage 1984 und GuTSCHE, Mitteleuropaplanungen 1972. Zu den wilhelminischen Mitteleuropaplänen auch THEINER, „Mitteleuropa“-Pläne 1984.

22 NaumanN, Schriften zum Mitteleuropaproblem, in: Ders., Werke, Bd. 4, 1964, S. 374-977. Die geistig-politische Werksgeschichte faßt zusammen: VILLAIN, Mitteleuropakonzeption Friedrich Naumanns 1977.

${ }^{23}$ Vgl. GuTsCHE, Mitteleuropaplanungen 1972, S. 537. 
Machtgefüge ${ }^{24}$. Schon auf der Flottenkonferenz von Washington 1922 manifestierte sich das Ende der britischen Ära in der Angleichung der US-amerikanischen Flottenstärke an die britische ${ }^{25}$. Unmittelbare Folge dieser Entwicklung war die Ablösung des weltpolitischen Zentrums Europa durch ein politisch-ideologisches Spannungsfeld: Der Aufstieg der außereuropäischen Weltmächte USA und UdSSR trug seit der russischen Oktoberrevolution den Nukleus einer globalen Dichotomie in sich, auch wenn beide Staaten zunächst noch einmal auf sich selbst zurückgeworfen wurden: Die Westmacht verfolgte einen gemäßigt isolationistischen Kurs, während ihr östlicher $\mathrm{Ge}-$ genpol in einen Bürgerkrieg verwickelt wurde ${ }^{26}$. Insoweit ist Lipgens' Einschätzung zuzustimmen: „Die eine Macht wollte, die andere konnte noch keine Hegemonialrolle in Europa übernehmen. Europa war noch einmal eine Frist gegeben." 27

Die faktische Überwindung des seit dem 19. Jahrhundert streng national verstandenen Souveränitätsprinzips wurde bezeichnenderweise repräsentiert durch die beiden neuen Weltmächte. Sowohl die USA als führende Weltwirtschaftsmacht und entscheidende militärische Kraft im Ersten Weltkrieg als auch die UdSSR mit ihrem neuen, auf Weltrevolution ausgerichteten Gesellschaftsmodell sprengten beide auf ihre Weise die bisherigen Vorstellungen von Staat und Gesellschaft. Die europäischen Staaten dagegen zogen sich ins Schneckenhaus nationalstaatlicher Souveränität zurück. Dieses Beharren Europas auf tradierten Strukturen erwies sich schnell als gefährlicher Anachronismus, der Europa wirtschaftlich, politisch, gesellschaftlich und in der völkerrechtlichen Entwicklung ins Hintertreffen geraten ließ. Der Versuch europäischer Politiker, die faktische Niederlage des auf seine imperialistische Spitze getriebenen Nationalismus durch eine erneute emotionale Überhöhung des Nationalstaates zu überwinden, blieb ein müdes revirement der Maximen des ideologisierten Weltkrieges und sollte schon bald fehlschlagen.

Der vielfache Bruch des Jahres 1918 schuf ein prinzipiell verändertes Bedingungsfeld für die Gestaltung eines neuen Europas und versah den nun wieder populären Europagedanken mit einer „neuartige[n] Dringlichkeit“28.

${ }^{24}$ Etwas überspitzt, in der Sache aber völlig korrekt bezeichnet GREwE, Völkerrechtsgeschichte 21988, S. 679 die „Brechung der überragenden alleinigen Vormachtstellung Englands" als das "weltpolitisch wichtigste Ergebnis des ersten Weltkrieges“.

${ }_{25}$ Die in Washington vereinbarte Schlachtschiff-Kontingentierung von 5:5:3:1,5:1,5 (Großbritannien, USA, Japan, Frankreich, Italien) löste den bisherigen Zweimächtestandard (Überlegenheit der britischen Flotte über die beiden folgenden Flottenkontingente) $\mathrm{ab}$ und blieb auch auf den folgenden Flottenkonferenzen in Washington 1929 sowie in London 1930 und 1936 unangetastet; vgl. GREWE, Völkerrechtsgeschichte ${ }^{2} 1988$, S. $680 \mathrm{f}$.

26 Die verbreitete Einschätzung eines rücksichtslosen US-Isolationismus ist von KOLB, Weimarer Republik 52000, S. 62 zutreffend als „ein der Korrektur bedürftiges Klischee" zurechtgerückt worden.

27 LIPGENS, Anfänge 1977, S. 3.

28 LOTH, Weg nach Europa ${ }^{3} 1996$, S. 10. 
In den folgenden Europadebatten standen neben der jetzt drängenden Notwendigkeit einer europäischen Wirtschaftseinheit gegen die USA insbesondere die Institutionalisierung des Friedens, die Modernisierung des Souveränitätsprinzips und der Antibolschewismus als neue Motive im Vordergrund europäischer Einigungsbestrebungen. Fortan bestimmten die neuen Motive nicht nur die ideengeschichtliche Entwicklung, sondern auch die Realität europäischer Politik, beginnend mit den Pariser Friedensverhandlungen. Damit war der Grundstein gelegt für den Europadiskurs im deutschen Exil. In den Jahren nach 1918 wurde die realpolitische Reibungsfläche des politischen Exils aufgezogen - und darin liegt die eigentliche Bedeutung der europäischen Zwischenkriegszeit für das Verständnis der Europakonzeptionen im Exil. Einige zentrale Elemente des späteren Diskurses wie der Antibolschewismus oder die Entdeckung föderalistischer Lösungen waren schon in der Zwischenkriegszeit virulent. Doch hatten Überlegungen zu einer europäischen Einigung in den Jahren von 1918 bis 1933 keinerlei Verwirklichungschance, wie Tucholskys zynische Beschreibung von 1932 veranschaulicht:

„Da liegt Europa. Wie sieht es aus?

Wie ein bunt angestrichnes Irrenhaus. [...]

Wir? Zollhaus, Grenzpfahl und Einfuhrschein:

Wir lassen nicht das geringste herein.

Wir nicht. Wir haben ein Ideal:

Wir hungern. Aber streng national.

Fahnen und Hymnen an allen Ecken.

Europa? Europa soll doch verrecken!

Und wenn alles der Pleite entgegentreibt:

daß nur die Nation erhalten bleibt. [...]

Die Nation ist das achte Sakrament - !

Gott segne diesen Kontinent. ${ }^{29}$

\section{Gebrochene Strukturen - die Versailler Friedensordnung}

Zurecht erinnert Kolb daran, daß das „Mächtesystem der Zwischenkriegszeit [...] seine maßgebliche Prägung durch Verlauf, Ergebnisse und Folgewirkungen der Pariser Friedenskonferenz" 30 erhielt. Mit dem Vertrag von Versailles versuchte man vergeblich, das Ende einer Epoche zu besiegeln und gleichzeitig eine neue völkerrechtliche Ordnung zu institutionalisieren. Das spätere politische Exil beschäftigte sich bis 1945 durchgängig intensiv mit dem Versailler Vertrag und konzentrierte sich vorwiegend auf drei Ergebnisse von 1919: die in den Vertragsteilen II und III vereinbarten neuen Grenzen als Ausfluß des Selbstbestimmungsrechts der Völker; die Gründung des Völker-

29 TuchOlsky, Europa (1932), abgedruckt in: DeRS., Zwischen Gestern und Morgen 1952, S. 177.

${ }^{30}$ KOLB, Weimarer Republik 52000, S. 195. 
bundes (Teil I des Vertrages) als erster Versuch einer langfristigen Friedenssicherung ${ }^{31}$ und der „Kriegsschuldparagraph“ Art. 231 VV als Rechtsgrundlage für die Reparationsforderungen. Bedingt durch den ausgeprägten Kompromißcharakter des Versailler Vertragswerks und die interalliierten Differenzen ${ }^{32}$ trugen die Ergebnisse der Pariser Friedensverhandlungen von der ersten Stunde an schwer an einigen strukturellen Brüchen, die hier in sechs Punkten dargestellt werden sollen.

Zunächst litt die Zwischenkriegszeit international an der 1919 ausgesparten abschließenden Regelung der Reparationsfrage, deren Höhe erst auf der Konferenz von Boulogne am 21. Juni 1920 erstmalig festgelegt wurde, um in der Folgezeit ständig revidiert zu werden. Betrachtet man zudem die vorgenommene Verknüpfung der Reparationsregelung mit der Rückzahlung der alliierten Kriegsschulden, ist in der Retrospektive die tatsächlich eingetretene Verzögerung der wirtschaftlichen Erholung Europas leicht nachvollziehbar. Zutreffend ist auf die in der Reparationsfrage schlummernde permanente Brandgefahr für die gesamte Retablierung des internationalen Staatensystems hingewiesen worden: „Die wirtschaftlichen und politischen Reorganisierungsversuche in Europa nach 1919 hingen aufs engste zusammen mit der Regelung der Reparationen, von hier gingen darüber hinaus - schon wegen der unumgänglichen Beteiligung der USA als stärkster Wirtschaftsmacht der Welt - entscheidende Impulse auf die Entwicklung des internationalen Systems der Nachkriegszeit aus." 33

Ein weiterer Stein des Anstoßes war die inkonsequente Handhabung des Selbstbestimmungsrechtes der Völker, das der Versailler Vertrag in der Gestalt von 20000 zusätzlichen Grenzkilometern manifestiert hatte. So stieß die Teilung Oberschlesiens entgegen der Abstimmungsmehrheit von $60 \%$ auf Unverständnis. Schnell wurde deutlich, daß das Schlagwort der völkerrechtlichen Selbstbestimmung als Arkanum des europäischen Friedens zutiefst ungeeignet war. Denn statt die Nationalismen zu überwinden, bot es den Verfechtern eines strengen Souveränitätsverständnisses ein ideales Argument in der Auseinandersetzung mit föderalistischen und internationalistischen Tendenzen. Nationale Rückbesinnung fand verständlicherweise auch bei den durch die Versailler Friedensordnung neu geschaffenen Staaten großen Anklang. Doch waren weder die neuen Kleinstaaten im Osten noch die südosteuropäische Neugründung Jugoslawien geeignet, das überkommene Problem der multiethnischen und religiösen Koexistenzen zu lösen. Im Ergebnis versagte der Lösungsansatz kleiner Nationaleinheiten ebenso wie der der

31 Vgl. dazu DÚlfFer, Jalta 21999, S. 38.

32 KOLB, Weimarer Republik 52000, S. 23 unterstreicht, daß die eigentlichen Konfliktlinien nicht $\mathrm{zw}$ wschen Siegern und Besiegten, sondern innerhalb der Siegerkoalition verliefen.

${ }^{33}$ Ibid. S. 200. 
vorherigen Vielvölkerstaaten Österreich-Ungarns und des Osmanischen Reiches ${ }^{34}$.

Neben der Strukturschwäche des Völkerbundes, auf die später einzugehen sein wird, kennzeichnete die Versailler Friedensordnung die Unsicherheit im Umgang mit der sozialpolitischen Sprengkraft des Bolschewismus. Schon die Pariser Friedensverhandlungen wurden indirekt von antibolschewistischen Strömungen beeinflußt ${ }^{35}$. Das alleinige Abstellen auf eine antibolschewistische Argumentations- und Motivationslinie der Alliierten in Paris dagegen ist in seiner Monokausalität kaum haltbar, wenngleich der Hinweis tendenziell richtig und notwendig ist ${ }^{36}$. Die Angst der Verhandlungsteilnehmer vor einer „Bolschewisierung“ Europas muß wohl eher als Kern der Konkurrenz zwischen zwei unterschiedlichen Gesellschaftsentwürfen interpretiert werden, die später zu machtpolitischer Bedeutung gelangten ${ }^{37}$.

Schließlich sind die erheblichen interalliierten Interessengegensätze als Strukturschwäche des Versailler Vertrages zu benennen, da sie ihm von Beginn an den Stempel eines als Gesamtwerk wenig überzeugenden Kompromißfriedens aufdrückten. Während Clemenceau eine dauerhafte Schwächung Deutschlands zugunsten eines franko-hegemonialen cordon sanitaire wünschte ${ }^{38}$, übernahm der amerikanische Präsident Wilson mit der Idee des Völkerbundes die Rolle des Visionärs. In der Tradition der kontinentalen checks and balances wandte sich auch Großbritannien gegen eine zu deutliche Schwächung Deutschlands, um französischen Hegemonialplänen den Boden zu entziehen. Zudem wurde in der Erhaltung Deutschlands ein effektiver Puffer gegen den als neue Ostgefahr ausgemachten Bolschewismus gesehen ${ }^{39}$.

Von Anfang an war der Versailler Vertrag von allen Seiten, auch in den Siegerländern, harscher Kritik ausgesetzt ${ }^{40}$, und in Deutschland entwickelte

${ }^{34}$ Bei DÜLFFER, Jalta ${ }^{2} 1999$, S. 36 findet sich die mißverständliche Formulierung, das russische Zarenreich sei als supranationales Staatsgebilde zerschlagen worden. Tatsächlich war dieses „supranationale“ Staatsgebilde schon 1922 mit der Errichtung der UdSSR wiedererstanden.

${ }^{35}$ Dies wird mittlerweile nicht mehr bestritten, vgl. z. B. DÜLFFER, Jalta 21999, S. 36; KOLB, Weimarer Republik 52000, S. 24.

36 Vgl. aber MAYER, Politics and Diplomacy of Peacemaking 1967. Zum Streitstand vgl. KoLB, Weimarer Republik 52000, S. 198 f.

37 Vgl. DüLFFER, Jalta 21999, S. 37. Ähnlich argumentiert KOLB, Weimarer Republik 52000, S. 24, der den Antibolschewismus als omnipräsenten „Generalbaß" der Friedenskonferenz sieht; vgl. auch KRÜGER, Deutscher Nationalismus und europäische Verständigung 1983, S. 516.

${ }^{38}$ Die bei der feierlichen Überreichung des Vertrags gegenüber der deutschen Delegation geäußerten Worte: „Die Stunde der Abrechnung ist da." veranschaulichen die grundlegend unterschiedlichen Zielsetzungen, die in den Verhandlungen nur mühsam auf eine gemeinsame Formel hatten gebracht werden können; zit. n. Möller, Europa zwischen den Weltkriegen 1998, S. 24.

${ }^{39} \mathrm{Zu}$ den alliierten Verhandlungspositionen vgl. auch КОLB, Weimarer Republik 52000, S. $27 \mathrm{f}$.

40 So auch DÜLFFER, Jalta 21999 , S. 36. 
sich der Mythos vom „Versailler Schmachfrieden“ zur „kollektive[n] Selbst-

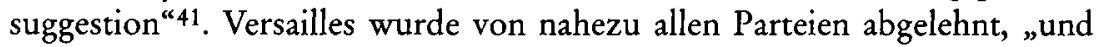
zwar mit emotionaler $\mathrm{Kraft}^{\prime 42}$ - zu lange hatte man sich der illusionären Hoffnung auf einen rücksichtsvollen „Wilson-Frieden“ hingegeben ${ }^{43}$. Dennoch darf nicht übersehen werden, daß im Versailler Vertrag durchaus vielversprechende Ansätze zu einem europäischen Neuanfang sowohl in friedensichernder als auch in wirtschaftlicher Hinsicht angelegt waren, die in den folgenden Jahren - wenn auch in ungenügendem und durch vielschichtige Hindernisse reduziertem Umfang - aufgenommen wurden. Dies gilt selbst in Anbetracht der Tatsache, daß sich Deutschland in der Zwischenkriegszeit und nicht erst seit 1930 - zur „revisionistischen Macht par excellence "44 aufschwang. Das Hauptproblem war die Ungeduld der Handelnden und die nur langsam mahlenden Mühlen kollektiver Wahrnehmungsprozesse: „,Versailles' als positiver Ausgangspunkt für eine neue europäische Ordnung, nicht als negatives Menetekel hatte also zeitweilig eine Chance - aber sie brauchte Zeit. Vertrauen mußte wachsen und wirken, um die mentalen Folgen des Krieges zu überwinden." 45

Im Ergebnis bleibt festzuhalten, daß der Friedensvertrag von Versailles dem besiegten Deutschland zwar harte Bedingungen auferlegte, zugleich aber ausdrücklich eine friedliche Revision zuließ und über den grundsätzlich intendierten Weg der Völkerverständigung einen baldigen Wiederaufbau Deutschlands zu einer europäischen Wirtschafts- und Politikmacht durchaus ermöglicht hätte ${ }^{46}$. Doch reagierte man noch im Exil mit blankem Unverständnis auf die Tatsache, daß gerade den demokratisch legitimierten Politikern der Weimarer Republik Zugeständnisse verweigert worden waren ${ }^{47}$, die den aggressiv fordernd auftretenden nationalkonservativen und schließlich nationalsozialistischen Politikern schließlich gewährt wurden. Dies veranschaulicht, daß einige konstruktive Elemente der Versailler Friedensordnung tatsächlich zukunftsweisende Strukturen bargen, die jedoch an den interalliierten Differenzen, der fehlenden internationalen Vertrauensbasis sowie der wachsenden Ungeduld deutscher Politiker scheiterten.

41 Ibid. S. 39.

42 Ibid. S. 40.

${ }_{43}$ Vgl. KOLB, Weimarer Republik 52000, S. 32.

44 Ibid. S. 34.

45 DülfFer, Jalta 21999, S. 43. KolB, Weimarer Republik 52000, S. 23 dagegen zeichnet das Vertragswerk selbst in dunkleren Farben, da er stärker aus der Retrospektive urteilt: „Den auf der Pariser Friedenskonferenz 1919 versammelten Staatsmännern ist es nicht gelungen, nach dem Ende des Weltkrieges eine einigermaßen stabile europäische Friedensordnung oder ein Weltfriedenssystem zu errichten, das die Gewähr wenigstens relativer Dauer zu bieten schien." Doch findet sich bei KoLB, ibid. S. 197 auch der ausdrückliche Hinweis auf die "recht weitgehende historische Rehabilitierung der ,Friedensmacher" von 1919“.

46 Vgl. Dülffer, Jalta 21999, S. 42; KOLB, Weimarer Republik 52000, S. 35.

47 Vgl. ibid. S. 35. 


\section{Machtloses Instrumentarium - der Völkerbund}

Diese Brechung der Strukturen spiegelte sich besonders deutlich im Völkerbund wider als der internationalen Organisation, die eine bisher nicht gekannte Weltordnung des Rechts einführen, überwachen und sichern sollte. Wilsons Idee zielte auf die Errichtung einer Weltfriedensordnung ab, die auf den Prinzipien des Völkerrechts - hier war das Selbstbestimmungsrecht der Völker der maßgebliche Kern ${ }^{48}$ - und der ausgleichenden Völkerverständigung basieren sollte. Unterstützung hatte Wilson unter anderem von dem südafrikanischen Ministerpräsidenten Smuts in einer Denkschrift vom Dezember 1918 erhalten ${ }^{49}$, zumal eine solche globale Neuordnung die Beseitigung der allseits beklagten und bis heute vielzitierten „zwischenstaatlichen Anarchie" versprach ${ }^{50}$. Die vornehmsten Aufgaben des Völkerbundes bestanden in der allgemeinen Abrüstung, der Etablierung eines Systems kollektiver Sicherheit und der Bereitstellung politischer Mechanismen zur friedlichen Konfliktbeilegung (Art. 8-17 VV) ${ }^{51}$. Die Satzung des Völkerbundes war als Teil I in die Friedensverträge integriert worden, so daß sie zusammen mit dem Versailler Vertrag am 10. Januar 1920 in Kraft trat. Schon im darauf folgenden Jahr erschien die deutsche Kommentierung der Satzung, die mit Walther Schücking und Hans Wehberg zwei herausragende Völkerrechtler und Pazifisten als Herausgeber aufweisen konnte ${ }^{52}$.

Doch worin lag die besondere Bedeutung der Völkerbund-Satzung? Sicherlich löste sie keine einzige der drängenden Territorialfragen, zumal Art. 10 die territoriale Integrität und politische Unabhängigkeit der Mitgliedstaaten für unantastbar erklärte. Somit entwickelte sich der Völkerbund schnell zum „Konservator des durch die Friedensverträge geschaffenen territorialen Status quo "53. Diese Rolle wurde auch nicht durch die Möglichkeit einer friedlichen Vertragsrevision nach Art. 19 VV relativiert, da diese die als Regelfall konzipierte Einstimmigkeit des Bundesversammlungsbeschlusses erforderte $^{54}$. Vielmehr ist die überragende Bedeutung der Völkerbund-Satzung als "historisches Dokument ersten Ranges" 55 in den übergreifenden Zielsetzungen zu sehen, die die Präambel treffend schildert: „to promote international co-operation and to achieve international peace and security by $[\ldots]$ the prescription of open, just and honourable relations between nations,

48 Vgl. BüLCK, Föderalismus 1964, S. 26.

49 Vgl. dazu KolB, Weimarer Republik 52000, S. 25.

50 Statt vieler LiPGENS, Anfänge 1977, S. 4.

51 Vgl. auch PfEIL, Völkerbund 1976, S. $53 \mathrm{f}$.

52 SCHÜCKING, WeHBERg (Hg.), Die Satzung des Völkerbunds ${ }^{2} 1924$.

53 KOLB, Weimarer Republik 52000, S. 26.

54 Vgl. PfEIL, Völkerbund 1976, S. 58. Dagegen ist die Einschätzung Pfeils, ibid. Anm. 31 sowie S. 147, die "praktische Nichtanwendung von Artikel 19 [habe] wesentlich zum Scheitern des Völkerbunds beigetragen“, zu weitgehend.

55 Ibid. S. 45. 
by the firm establishment of the understandings of international law as the actual rule of conduct among Governments, and by the maintenance of justice and a scrupulous respect for all treaty obligations in the dealings of organized peoples with one another"

Mit der Völkerbund-Satzung wurde erstmalig der Versuch unternommen, die Friedenssicherung auf einer übernationalen Ebene zu institutionalisieren und das Völkerrecht als verbindlichen, einheitlich ausgelegten Maßstab zugrunde zu legen. Der Internationale Gerichtshof in Den Haag stellte eine gemeinsame Institution der Rechtswahrung und des Rechtsschutzes dar, der in der Tat einige Streitigkeiten zwischen kleineren Staaten zu schlichten vermochte. Auseinandersetzungen der Großmächte wurden jedoch weiterhin an IGH und Völkerbund vorbei verhandelt.

Die internationale Vereinbarung eines Primats des Rechts brachte eine neue Qualität des Völkerrechts - eines Rechtsgebietes, das in besonderer Weise vom politischen Impetus abhängt und bis heute viel deutlicher unter dem Kuratel der Politik steht als das innerstaatliche Recht demokratischer Rechtsstaaten. Anders als 1945 bedeutete die Völkerbund-Satzung daher einen Wendepunkt in der Entwicklung des Völkerrechts ${ }^{57}$, der mit der Institutionalisierung der Konfliktbewältigung eine stärkere Verrechtlichung und die Verankerung völkerrechtlicher Nova wie der Haager Schiedsgerichtsbarkeit und des angelsächsischen $H u e$ and $C r y$ nach sich zog 58 und das Recht der internationalen Organisationen als eigenständiges Element des Völkerrechts begründete ${ }^{59}$. Damit hatte der europäische Kontinent auch als System kollektiver Sicherheit eine neue Qualitätsstufe erreicht, worin mit einiger Berechtigung die „eigentlich wegweisende Idee der Friedenskonferenz ${ }^{60}$ gesehen worden ist.

Mithin stellte der Völkerbund nicht nur den Versuch dar, das Europäische Konzert auf der globalen Ebene zu organisieren ${ }^{61}$, sondern darüber hinaus auch qualitativ entscheidend weiterzuentwickeln. Vor diesem Hintergrund ist die Tatsache bedeutsam, daß drei der ursprünglich vier ständigen Mitglieder europäische Staaten waren: Großbritannien, Frankreich und Italien. Hartwig Bülck interpretierte diese Konstellation 1964 noch als „föderativ erweiterte Kollektivhegemonie"62, doch kann dieser Einschätzung weder inhaltlich noch terminologisch gefolgt werden. Schon 1919 war die Schwäche der europäischen Staaten einschließlich Großbritanniens und Frankreichs erkennbar, und die Abhängigkeit des Völkerbundes von der Entwicklung des außereuropäischen Spannungsfeldes zwischen den USA und der UdSSR so-

56 Zit. n. ibid. S. 46.

57 Vgl. GREwE, Völkerrechtsgeschichte 21988, S. 679.

58 Vgl. BülCK, Föderalismus 1964, S. 27.

59 BUERGENTHAL et al., Völkerrecht 22000, S. 14.

60 KOLB, Weimarer Republik 52000, S. 26.

61 So BülCK, Föderalismus 1964, S. 26.

62 Ibid. 
wie der weltwirtschaftlichen Führungsposition der USA ließen keinen Raum für eine europäische „Kollektivhegemonie“ - ein Begriff, der schwer an der ihm innewohnenden Widersprüchlichkeit zu tragen hat. Auch die Behauptung einer „föderativen“ Erweiterung des Europäischen Konzerts ist kaum nachvollziehbar und wohl nur unter Zugrundelegung des weiten Föderalismusbegriffs verständlich, von dem Bülck in seiner Untersuchung ausgeht und demzufolge schon das Europäische Konzert des 19. Jahrhunderts einen föderativen Charakter aufwies. Der bisweilen exzessive Rückgriff auf den Terminus „föderativ“ gerät bei Bülck zu einer Verdunkelung der Grenzen zwischen einer bloßen zwischenstaatlichen Kooperation einerseits und einer auf dem Grundsatz der Staatengleichheit beruhenden Föderation andererseits. Ob innerhalb einer solchen Föderation einzelne Staaten oder Staatengruppen stärkeren Einfluß ausüben als andere - wovon regelmäßig ausgegangen werden darf -, ist keine Frage der föderativen Struktur, sondern schlicht der politischen Machtverteilung intra muros ${ }^{63}$.

Die Konstruktion des Völkerbundes wies einige gravierende Schwächen auf, die ihn bald aus dem Zentrum der internationalen Aufmerksamkeit entfernten. Die entscheidende Strukturschwäche ist in der Diskrepanz zwischen dem universalistischen Ansatz und der europäisch zugeschnittenen Mitgliederstruktur zu sehen, die zu keinem Zeitpunkt eine wahrhaft globale Ausrichtung widerzuspiegeln vermocht hat ${ }^{64}$. Dies veranschaulicht die wechselnde Zusammensetzung der ständigen Mitglieder: Von den entscheidenden außereuropäischen Staaten weigerten sich die USA von Anfang an, dem Völkerbund beizutreten, während der Sowjetunion die Mitgliedschaft erst 1934 gewährt wurde - um sie ihr schon im Dezember 1939 wegen des Angriffs auf Finnland wieder zu entziehen. Als dritter außereuropäischer Machtfaktor schied Japan im März 1933 aus. Alle anderen übrigen ständigen Mitglieder waren europäische Staaten: Deutschland wurde als Kriegsverlierer erst 1926 aufgenommen 65 und trat schon im Oktober 1933 wieder aus. Und im Dezember 1937 trat Italien aus dem Völkerbund aus, so daß im Jahr des Kriegsbeginns 1939 bezeichnenderweise die beiden europäischen Großmächte Großbritannien und Frankreich übrig blieben ${ }^{66}$. In engem Zusammenhang

${ }^{63}$ Die völkerrechtliche Klassifizierung des Völkerbundes wurde schon früh diskutiert. Wie Bülck geht auch ZimmerN, The League of Nations and the Rule of Law 1936, von einer unmittelbaren Fortsetzung des Europäischen Konzerts aus, während HUBER, Die konstruktiven Grundlagen des Völkerbundsvertrages, in: Zeitschrift für Völkerrecht 12 (1920), S. $170 \mathrm{ff}$. den Völkerbund als Staatenverbindung sui generis ansieht.

6442 Gründungsmitglieder; bis 1937 kamen 21 hinzu, aber bis 1942 traten auch 20 Mitgliedstaaten wieder aus; vgl. PFEIL, Völkerbund 1976, S. $47 \mathrm{f}$.

65 Mit dem Austritt Japans und Deutschlands aus dem Völkerbund 1933 setzte eine weltumspannende Aggressionsdynamik ein, die sich schon in der Mandschurei-Krise angekündigt hatte und die Ohnmacht des Völkerbundes in der Krisenprävention offenbarte, sobald die Großmächte beteiligt waren; vgl. auch GREwE, Völkerrechtsgeschichte 21988 , S. 682.

66 Vgl. auch den Überblick bei MölLER, Europa zwischen den Weltkriegen 1998, S. 38. 
mit diesem gestörten Verhältnis zwischen Weltgeltungsanspruch und monokontinentaler Orientierung steht die verpaßte Gründung einer eigenen europäischen Regionalorganisation im Rahmen eines wirklich weltumspannenden Völkerbundes. Nur eine solche in Art. $21 \mathrm{VV}$ ausdrücklich zugelassene Konstellation hätte einen befriedigenden Ausgleich zwischen Deutschland und Frankreich ermöglichen können, der schon damals für den gesamteuropäischen Frieden grundlegend war. Diese verpaßte Chance gehörte „zweifellos zu den bedenklichsten Schwächen der Pariser Friedensordnung ${ }^{{ }^{677}}$. Mit Blick auf die späteren Exildiskussionen um einen neuen Völkerbund und eine Neuordnung Nachkriegseuropas verbietet sich daher eine Trennung der Europadiskussion von der Völkerbundsdiskussion im Exil, da beiden Anliegen derselbe Gedanke zugrunde lag und bis auf wenige Ausnahmen eine europäische Staatenverbindung allein im Rahmen eines übergeordneten Weltbundes erörtert wurde. Diese Verknüpfung war schon in Weimar verfochten worden und findet sich letztlich auch bei Briand, auch wenn sein Plan von 1930 hauptsächlich aus Gründen politischer Praktikabilität auf eine losgelöste Europa-Organisation verzichtete.

Ein gravierendes Manko war die politische Abstinenz der USA, die sich auf Druck der Legislative dem Völkerbund nicht anschlossen, obschon die Idee gerade von einem amerikanischen Präsidenten herrührte. Deshalb blieb der Völkerbund als politische Institution ebenso hinter der weltpolitischen Realität zurück wie das Völkerrecht mit diesem ersten ernsthaften Versuch seiner allgemeinverbindlichen Kodifizierung schon im Augenblick seines Inkrafttretens von der Wirklichkeit der tatsächlichen weltpolitischen Machtverteilung überholt war ${ }^{68}$. Darüber hinaus krankte der Völkerbund an der seiner ursprünglichen Aufgabe diametral entgegenstehenden Realpolitik. Frankreich versuchte bis 1924, den Versailler Vertrag zu revidieren, was sich auch in den nachfolgenden Jahren noch hemmend auswirkte ${ }^{69}$, während Großbritannien gesteigertes Interesse an einer möglichst baldigen wirtschaftlichen Reintegration Deutschlands zeigte. Folglich war der Völkerbund den einen grundsätzlich zu lasch, den anderen zu hart. Unverändert herrschte das Dogma nationalstaatlicher Macht- und Bündnispolitik vor, ungeachtet der fortschrittlicheren Völkerbund-Satzung ${ }^{70}$. Zudem trug die verspätete Einbindung des besiegten Deutschlands dem Völkerbund in der - namentlich deutschen - Bevölkerung den Ruf eines „Bundes der Siegerstaaten“71 ein und verstärkte die deutsche Rolle als Außenseiter unter den europäischen Orientierungsmächten.

Schließlich bot die Völkerbund-Satzung aufgrund ihres Charakters als politischer Kompromiß nur eingeschränktes Fortschrittspotential. Insbeson-

67 KRÜGER, Deutscher Nationalismus und europäische Verständigung 1983, S. 514.

68 Vgl. dazu GrEWE, Völkerrechtsgeschichte 21988, S. 681.

69 Vgl. DÜlfFer, Jalta 21999, S. 41.

70 So auch KOLB, Weimarer Republik 52000, S. 27.

71 Ibid. S. 26. 
dere das bloß relative Kriegsverbot statt einer ausdrücklichen Kriegsächtung und die bewußt schwach ausgestattete Exekutivgewalt sowie der Einstimmigkeitsgrundsatz ${ }^{72}$ ließen den Völkerbund als machtloses Instrumentarium innerhalb einer an vielfältigen Konfliktlinien gebrochenen Struktur erscheinen, das viele der ursprünglich vielversprechenden Neuansätze des Versailler Vertrages zu bald vergessenen Absichtserklärungen degradierte ${ }^{73}$. Hattenhauer ist der Ansicht, die Europäer seien "noch nicht so klug [gewesen] zu erkennen, daß auf dem alten Kontinent der Frieden nur durch Souveränitätsverzichte der Nationalstaaten und die Übertragung von Hoheitsrechten auf eine übernationale Macht zu erreichen war"74. Eine solche, die historische Perspektive zugunsten einer rein juristisch funktionalen Sichtweise ausblendende Betrachtung ist in ihrer moralisierenden Vereinfachung einer späteren Generation kaum haltbar. Vielmehr muß den damaligen Umständen Rechnung getragen werden, die - unmittelbar nach dem bis dato schrecklichsten Kriegserlebnis und in der allgemeinen Stimmung überhöhter Nationalismen - eine Beschneidung der nationalen Souveränität keineswegs als einzige Lösung der Spannungen erscheinen ließ. Dies gilt erst recht für die Übertragung von Hoheitsrechten auf eine mangels historischen Vorbildes kaum einschätzbare Organisation wie den Völkerbund, dem entscheidende Mächte überhaupt nicht angehörten ${ }^{75}$.

Der Völkerbund war in den Schwächen seiner eigenen Struktur gefangen und auf dem internationalen Parkett der Diplomatie nicht mehr, aber auch nicht weniger als ein ernsthafter Versuch langfristiger Friedenssicherung. Die Abstinenz der USA und die fehlende Beteiligung der UdSSR, eine in Teilen überzogene Demütigung Deutschlands und das allgemeine Festhalten an den Maßstäben traditioneller Machtpolitik verurteilten diesen Versuch zum Scheitern. Während Großbritannien in einer Mischung aus Ratlosigkeit und Tradition an der überkommenen Politik der checks and balances festhielt, ohne den eigenen Bedeutungsverlust wahrhaben zu wollen, war Frankreich zu schwach, um in Europa eine gestaltende Führungsrolle zu übernehmen. Darüber hinaus lagen keinerlei Pläne für die Gestaltung der spezifisch europäischen Angelegenheiten vor, obwohl Europa schon seit längerem einer

72 Ausgenommen von der Einstimmigkeitspflicht waren lediglich einige sekundäre Verfahrensfragen gemäß Art. $5 \mathrm{VV}$.

73 Vgl. zusammenfassend PFELL, Völkerbund 1976, S. 57.

${ }^{74}$ Hattenhauer, Europäische Rechtsgeschichte 1992, S. 668.

${ }^{75}$ Hierzu bemerkt HATTENHAUER, ibid. S. 670, daß der Völkerbund nicht daran gedacht habe, „im Wege richterlicher Rechtsfortbildung die Stellung des Bundes über seine ihm in der Satzung gezogenen Grenzen auszudehnen“. Eine solche Polemik verkennt jedoch völlig die historische Bedingtheit des Völkerbundes und urteilt ahistorisch unter Zugrundelegung heutiger Maßstäbe. Hier wird mit durchsichtigen Absichten die Kritik der heutigen Rechtswissenschaft an der Praxis des Europäischen Gerichtshofs, seine Zuständigkeitskompetenzen durch richterliche Rechtsfortbildung selbst zu erweitern, mit dem in diesem Fall recht dünnen Mantel der Geschichte bedeckt. 
Neuordnung harrte. Stattdessen definierte die Satzung des Völkerbundes einen globalen Geltungsanspruch, ohne sich für problemorientierte kleinere Einheiten, etwa einen europäischen Regionalbund, einzusetzen ${ }^{76}$. Mithin erlitt der Völkerbund das für jede internationale Organisation vernichtende Schicksal der schleichenden Entpolitisierung durch die wachsende Mißachtung seitens der Mitgliedstaaten. Anfangs blieb der Völkerbund „auf die Rolle eines moralischen Vermittlers beschränkt, bis die großen Mächte es vorzogen, ihre Streitfragen in direkten Verhandlungen unter sich zu erörtern" $" 77$. Die Folge war, daß die nationale Souveränität der Staaten unangetastet blieb, die traditionelle Machtstaatenpolitik unverändert fortgesetzt wurde ${ }^{78}$ und der riesige Verwaltungsapparat des Völkerbundes in den Augen vieler Zeitgenossen zu einer intergouvernementalen Einrichtung verkümmerte, dessen größtes Verdienst darin bestand, künftigen Europaplanern als Negativfolie ihrer Konzepte zu dienen. In der Retrospektive aber ist das Moment der Institutionalisierung eines Friedensmechanismus von höchster Bedeutung, das den Völkerbund in der historischen Dimension ungeachtet seiner unglücklichen Geschichte als eine „bahnbrechende Neuerung "79 erscheinen läßt. Losgelöst von der Frage realer Umsetzungserfolge führte das Moment der Institutionalisierung per se für die Völkerrechtsgeschichte zu einer Zäsur, die den Übergang zum "nachklassischen Völkerrecht" markier ${ }^{80}$. Als hervorstechendste Eigenschaft des "nachklassischen Völkerrechts" sollte sich in den folgenden Jahrzehnten die Verselbständigung des Völkerrechts vom Zivilisationsgedanken herausstellen, „ohne daß man allerdings in der Lage gewesen wäre, ihm eine neue, allgemeinverbindliche Wertordnung zugrunde zu legen" 81 .

Während der Völkerbund anfänglich zwar als vielversprechender Ansatz betrachtet wurde, wegen seiner Einbindung in den Versailler Vertrag und des fehlenden Vertrauens in seine Konfliktlösungskompetenz aber weder Eigenständigkeit noch Machtfülle besaß, war „Versailles“ eine Realität, die von vielen Seiten von Beginn an als zu bekämpfende Ordnung angesehen wurde. Doch sollten die tatsächlich errungenen Kompromisse, insbesondere die Gründung des Völkerbundes und der konstruktive Einfluß der US-amerika-

76 Vgl. KRÜGER, Deutscher Nationalismus und europäische Verständigung 1983, S. 514.

77 HatTEnHauer, Europäische Rechtsgeschichte 1992, S. 669.

78 Vgl. auch PFEIL, Völkerbund 1976, S. 51.

79 Ibid. S. 50.

80 GREWE, Völkerrechtsgeschichte ${ }^{2} 1988$, S. 679, 685. Das Urteil HATTENHAUERs, Europäische Rechtsgeschichte 1992, S. 669, der Völkerbund sei „nicht ohne rechtliche Bedeutung" gewesen, wird dem Zäsurcharakter der Gründung des Völkerbundes nicht gerecht.

81 GREwE, Völkerrechtsgeschichte 21988 , S. 690 et passim. Der Gedanke der Ablösung der Rechtssolidarität der Zivilisationsgesellschaften durch ein offenes, weltumspannendes Völkerrecht in der Zwischenkriegszeit ist eine der Hauptaussagen in Grewes Werk. 
nischen Forderungen nicht unterschätzt werden, ohne die eine solche Institution wohl kaum zustande gekommen wäre ${ }^{82}$.

Der Erste Weltkrieg provozierte drei oder zumindest in ihrer Intensität völlig neue Motivationsstränge in der bis dato eher akademisch behandelten Europafrage: die Institutionalisierung des Welt- und Kontinentalfriedens, die Errichtung einer europäischen Wirtschaftseinheit und die Verwirklichung einer antibolschewistischen Abwehr. Als Strukturelement einer mit diesen Aufgaben zu betrauenden Staatenverbindung rückte der Föderalismus erstmals in das Zentrum der Debatten. In der Zwischenkriegszeit konnte sich die Idee einer föderal geordneten europäischen Ebene ebensowenig durchsetzen wie das dreifache Motivbündel ${ }^{83}$ : „Es entstand trotz einiger Ansätze keine kraftvolle politische Bewegung zugunsten europäischer Zusammenarbeit und Integration." 84

In diesem Zusammenhang seien die rein pragmatisch motivierten Bemühungen der Wirtschaft ebenso erwähnt wie die eher visionären gesellschaftlichen Kontroversen um das Ideal eines geeinten europäischen Kontinents. Für die anhaltenden Rivalitäten zwischen Richard Graf Coudenhove-Kalergi sowie Wilhelm Heile und Alfred Nossig ist ein Tagebucheintrag Erich KochWesers, selbst Mitglied des Komitees für die Interessengemeinschaft europäischer Völker, bezeichnend: „Sehr viel Ärger habe ich in dieser Zeit von dem Zusammenstoß der Idee des Grafen Coudenhove mit den Bestrebungen Heile/Nossig gehabt. Es liegt in der Tat so, daß hier kleine Geister, denen es um ihr Brot zu tun ist, sich einen großen Gedanken angeeignet haben. ${ }^{185} \mathrm{Da}$ bei darf jedoch nicht übersehen werden, daß die Paneuropaidee einerseits und die Europavorstellungen der Heile-Nossig-Gruppe andererseits keineswegs kompatibel waren, so daß eine Fusion der Gruppen jenseits der persönlichen Konfliktlinien auch aus konzeptionellen Gründen kaum möglich gewesen wäre. Auch die Versuche der europäischen Wirtschaft, sich in engeren Kooperationen zusammenzuschließen, waren im Ergebnis nicht von Erfolg gekrönt. Zudem zielte insbesondere die deutsche Wirtschaft mehrheitlich auf die Errichtung einer liberalen Weltwirtschaft im Sinne der Free-Trade-Doktrin und stand der Idee eines auf den europäischen Kontinent begrenzten $\mathrm{Zu}$ sammenschlusses daher skeptisch bis ablehnend gegenüber ${ }^{86}$. Für die europäischen Überlegungen in Wirtschaft und Gesellschaft der Weimarer Repu-

82 Vgl. Kolb, Weimarer Republik ${ }^{5} 2000$, S. 198.

83 Zustimmend, aber nur auf den Aspekt fehlgeschlagener Friedensinstitutionalisierung abstellend LIPGENS, Anfänge 1977, S. 4.

84 KRÜGER, Europagedanke in der Weimarer Republik 1996, S. 25.

85 Tagebucheintragung vom 12.8. 1926, zit. n.: Holl, Europapolitik 1974, S.61, Anm. 85.

86 Insofern zutreffend die Feststellung von LIPGENS, Anfänge 1977, S. 5: „Alle Bemühungen der zwanziger Jahre um Wiederherstellung einer liberalen Weltwirtschaft blieben vergeblich, weil die europäischen Länder auf ihre sinkende Importfähigkeit auch untereinander mit Abwürgung des innereuropäischen Handels, mit Erhöhung der (durch Versailles um rund $20000 \mathrm{~km}$ vermehrten) Grenz- und Zollmauern reagierten." 
blik gilt gleichermaßen, daß sie mit Ausnahme der Paneuropaidee des Grafen Coudenhove-Kalergi für den Europadiskurs im politischen Exil der Jahre 1933-1945 nahezu einflußlos blieben.

Dagegen waren die politischen, völkerrechtlichen und institutionellen Ideenansätze der $Z$ wischenkriegszeit weiterhin virulent und prägten die zeitgenössische Wahrnehmung der Europadebatten. Diese Ansätze eines - vorerst allerdings gründlich gescheiterten - Europe vécue wurden von deutscher Seite ins Exil hinübergerettet, um dort, befreit von den Zwängen und Rücksichtnahmen der Alltagspolitik, in zahlreichen Modellen eines Europe pensée modifiziert und weiterentwickelt zu werden.

Mit der Umsetzung einer europäischen Einigung war die Zwischenkriegszeit erkennbar überfordert - nicht nur wegen der politischen Partikularinteressen, des Wiederauflebens von Nationalismus und Revisionismus, sondern auch deshalb, weil Europa als gemeinsames, konsensfähiges Wertesystem nicht etabliert, in vielerlei Hinsicht noch nicht einmal definiert war. Somit konnte die Zwischenkriegszeit - und erst recht nicht die seit ihrer Gründung um innenpolitische Existenz und außenpolitische Akzeptanz ringende Weimarer Republik - keine langfristigen Lösungen für Europa entwerfen, geschweige denn realisieren ${ }^{87}$.

Unweigerlich übten der Versailler Vertrag und die Idee des Völkerbundes eine zwiespältige, aber gerade deshalb gestalterisch wirkende Anziehungskraft auf die Debatten des politischen Exils um eine europäische Neuordnung aus. Sie prägten den Bewußtseins- und Erfahrungshorizont der späteren Emigranten und spielten hinein in die Ausgangspositionen der außenpolitischen Debatten im Exil. Somit ist ohne die Kenntnis der zeitgenössischen Einschätzung des Versailler Vertrages und seines wichtigsten Werkzeuges ein Verständnis der Europakonzeptionen des Exils nur bedingt möglich. Vor diesem Hintergrund kann schon jetzt festgehalten werden, was Hubertus Prinz zu Löwenstein noch 1939 äußerte: „Es bleibt, daß der Gedanke einer Weltordnung des Rechtes nicht nur ausgesprochen wurde, sondern eine Verwirklichung fand, allem Widerstand und allem sogenannten Versagen zum Trotze, und daß dies von nun an nicht mehr hinwegzudenken ist. ${ }^{" 88}$

87 Vgl. KRÜGER, Europagedanke in der Weimarer Republik 1996, S. 28.

${ }^{88}$ LÖWENSTEIN, Die Zukunft Europas. Eine Rede, gehalten am 3.11. 1939 vor dem "International Relations Club" in San Diego, Kalifornien, 18seitiges Typoskript, ANF, F7 15126, M. 2. 